\title{
Communications Resources Management for Advanced Telematics Applications
}

\author{
Ratish J. Punnoose, Richard S. Tseng, Stanley Wang, Pavel V. Nikitin, T.E. Schlesinger, \\ and Daniel D. Stancil \\ CMU/GM Satellite Research Laboratory \\ Department of Electrical and Computer Engineering \\ Carnegie Mellon University, Pittsburgh PA 15213
}

\begin{abstract}
Vehicles in the future will have significant increases in capability and demands for wireless communications resources. Applications include vehicle status and maintenance information, navigation information, entertainment, and concierge services. To meet these needs, the vehicle must have the capability to allocate and prioritize communications resources in response to the needs of applications. We propose a strategy for a communications resources architecture to address these needs.
\end{abstract}

Keywords-Telematics, Wireless, Communications.

\section{INTRODUCTION}

Future telematics applications will bring intelligent computing to vehicles [1]. Various applications for safety, remote diagnostics, maintenance, and entertainment will be embedded into vehicles [2]. Different telematic applications will have different communications requirements, so external communication networks with diverse properties will be needed. Further, the wireless network architecture must be designed to support vehicle mobility [3].

Applications currently being rolled out to "pump information while pumping gas" (Ten Square Corporation www.tensquare.com) require a high-speed localized wireless connection. Emergency diagnosis requires a highly available connection but it could be low-speed. These applications will need a variety of communication mechanisms. Multiple telematic applications will be in use on the vehicle at the same time. It is not unreasonable to expect software agents on the computer to perform many different tasks simultaneously such as location-dependent content delivery, multimedia access, emergency message access, and intelligent path routing using traffic information and road conditions.

These software agents that perform different tasks may still need to access the same communications resources. The existence of a resource manager to allow uniform access through standard interfaces will simplify application development and support.

We can envision different forms of communications ser- vices that might be provided on a vehicle.

- Broadcast reception : Entertainment and information applications may need to access broadcast devices such as AM and FM radios, Digital Audio Broadcast, Television etc.

- Position : Map routing and proximity detection agents need current position and velocity information.

- Voice : Cellular/PCS calls require dedicated voice connections.

- Data: In general, multiple options for data connections such as in-vehicle short-range, high-speed out-of-vehicle, and slow speed long range data may be available. These data connections may have differing costs, bandwidths and ranges of operation. Software agents must be capable of requesting connections with varying Quality-of-Service requirements.

The software applications on the vehicle will have rich interaction with each other. As an example, the vehicle audio-visual system will be tightly integrated with driver communications. For example, when the driver receives a cellular call, the music volume in the automobile will automatically be decreased. A well integrated telematic system must support such inter-agent communication.

A resource manager should provide basic and structured access to available resources for all applications, and more sophisticated services to resource-manager aware applications. For example, a software entertainment agent can choose to provide radio service using a combination of FM broadcast and streaming audio over the Internet. The choice of access may depend on the location, availability, cost, and other parameters.

\section{System TeStBed}

A test-bed for the development of future telematics applications has been implemented in a vehicle. The current implementation uses off the shelf computers with various peripherals that can be operated under software control (Fig 1).

The vehicle has also been connected with a range of 


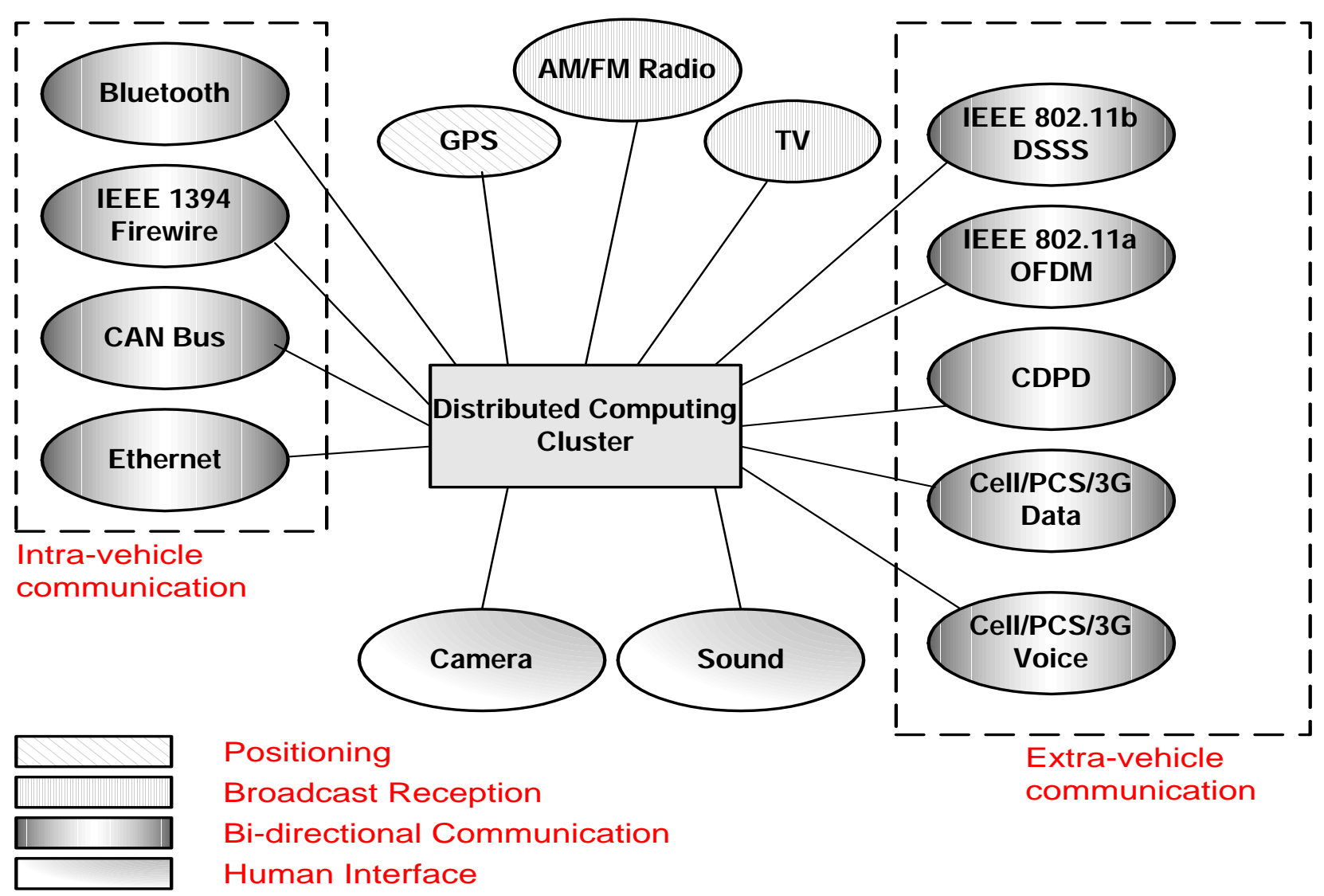

Fig. 1. System Architecture

\begin{tabular}{||r|l||}
\hline \hline Frequencies & Usage \\
\hline \hline $535-1605 \mathrm{kHz}$ & AM Radio \\
\hline $54-72 \mathrm{MHz}$ & TV Channels 2-4 \\
\hline $88-108 \mathrm{MHz}$ & FM Radio \\
\hline $174-216 \mathrm{Mhz}$ & TV Channels 7-13 \\
\hline $512-608 \mathrm{MHz}$ & TV Channels 21-36 \\
\hline $614-806 \mathrm{MHz}$ & TV Channels 38-69 \\
\hline $806-902 \mathrm{MHz}$ & AMPS, CDPD \\
\hline $1574-1576 \mathrm{MHz}$ & GPS L1 \\
\hline $2400-2484 \mathrm{MHz}$ & $\begin{array}{l}\text { IEEE 802.11, Bluetooth, } \\
\text { OFDM modem }\end{array}$ \\
\hline \hline
\end{tabular}

TABLE I

RF SPECTRUM OCCUPIED BY VEHICLE SYSTEMS

communications options. The current system has computer controlled television and FM radio capabilities. It also includes myriad wireless network connections (Table I).

- Bluetooth : A Bluetooth interface is provided for invehicle communication with devices such as PDAs.

- 802.11b DSSS : A high-speed 802.11b (Direct Sequence
Spread Spectrum) interface is also provided for internal or external communications.

- OFDM : A high-speed Orthogonal Frequency Division Modulation modem is also included for longer range highbandwidth multimedia communications. This represents 802.11a devices that will operate in the ITS band.

- GPS : A Global-Positioning Device that is capable of accepting differential corrections is also a part of the system. This is essential for map routing and delivery of location-dependent content.

- CDPD : A Cellular Digital Packet Data modem is included. This is a low-speed connection but uses the current wireless infrastructure and therefore has good coverage.

- AMPS cell phone: AMPS service is widely available through Northern America and provides a good fallback for voice service.

- Television and AM/FM radio : Radio service is essential since it is widely found in current vehicles. Television, though not as prevalent today, will become more so as entertainment services move into the vehicle.

The architecture at present represents a sampling of currently available technology but can be generalized to accomodate future systems. 


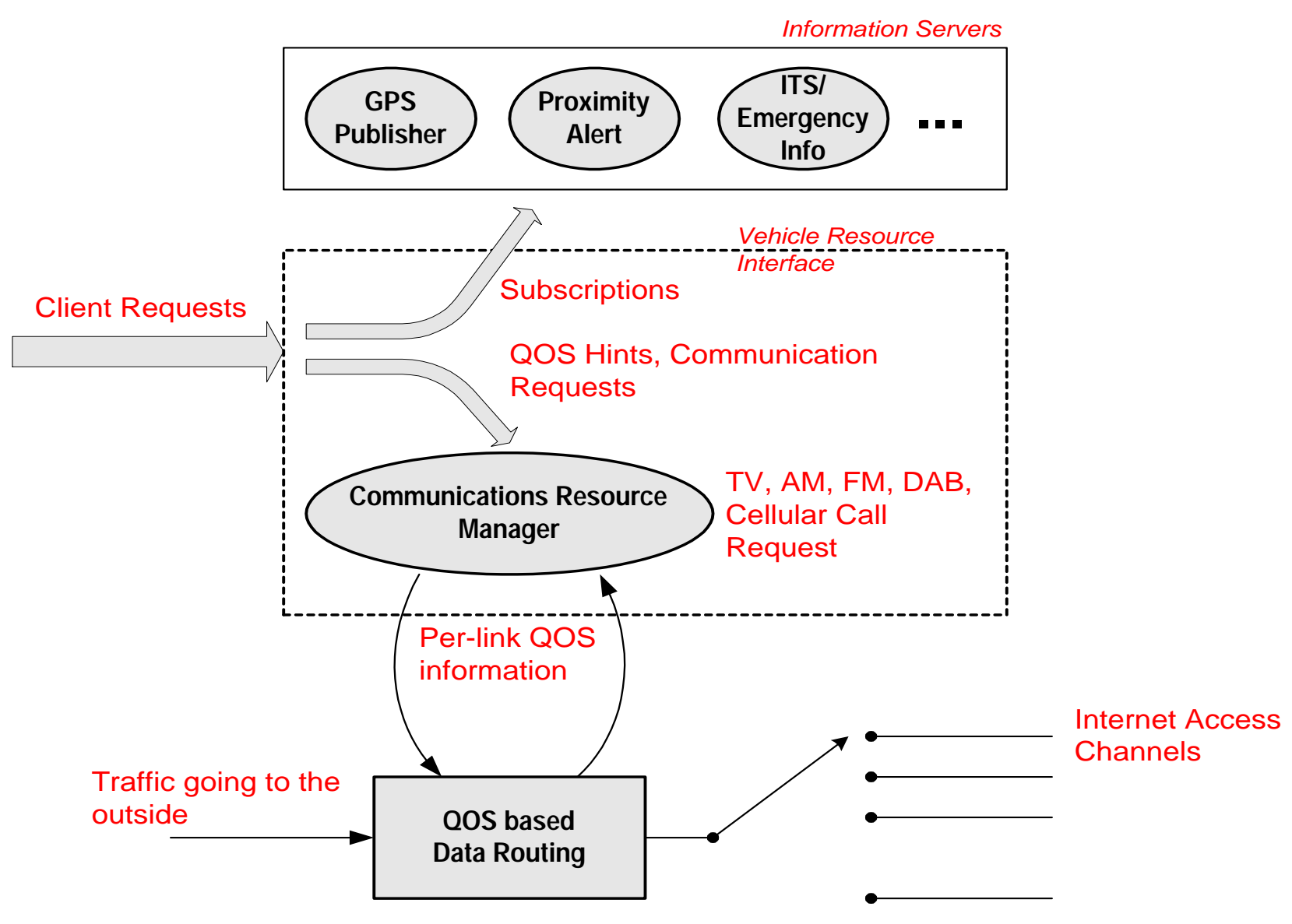

Fig. 2. Resource Manager

\section{COMmunicAtions Architecture}

A vehicle system with a variety of communications infrastructure also needs a standardized interface for software agents to access the communications resources. The vehicle computing system can be a distributed system and the operation of the resource manager can itself be distributed. The architecture of the resource manager is illustrated in Fig 2. The architecture supports mechanisms for internal and external communications.

\section{A. External Communications}

Communications between the in-vehicle components and the exterior passes through a router gateway. This routing module chooses the appropriate external communication method to use for data connections depending on parameters such as cost, availability, and QoS requirements of the applications using them. The resource manager interacts with this routing module to advise it of the QoS requirements of applications.

\section{B. Internal Communications}

Inter-agent communication within the vehicle has to be flexible to support the addition of new software agents. It is advantageous to use a model that allows the transfer of data without strict pre-definition. A publisher-subscriber model [4] can provide this. The key idea is that all agents that tap into this infrastructure can be publishers of information or subscribers of information or both. The actual information being published is transparent to the underlying infrastructure which merely transports it from the publishers to the subscribers. Publishers post information on logical channels. Other agents can be subscribers of this information. For example, there might be a software agent that interfaces with the GPS device and publishes positioning information. Other software agents that need positioning information can subscribe to this. The publishsubscribe infrastructure conveys information from the publisher to the subscribers transparently. This has the following advantages:

- Having various information servers separate from the resource manager simplifies the design and increases the reliability of the resource manager.

- It also allows for easy addition of more information servers without any modification to the basic architecture.

- The distributed nature allows nodes to join and leave the publish-subscribe domain dynamically. 


\begin{tabular}{|c|c|c|c|c|c|c|}
\hline $\begin{array}{c}\text { Network } \\
\text { Header }\end{array}$ & Version & $\begin{array}{c}\text { Connection } \\
\text { Identifer }\end{array}$ & $\begin{array}{c}\text { Request } \\
\text { Type }\end{array}$ & $\begin{array}{c}\text { Request } \\
\text { Subtype }\end{array}$ & Length & Request Specific Data \\
\hline
\end{tabular}

Fig. 3. API Packet Structure

- This decouples applications in the sense that publishers do not have to keep track of subscribers. The infrastructure takes care of these connection and logistical details.

\section{Interface to the Resource Manager}

The resource manager itself can be a single or distributed entity. Client applications communicate with the resource manager using network packets. Therefore the interface to the resource manager can be on the same processor as the client application or on a different machine within the vehicle. The only information the client has to have is the location of the resource manager. The current implementation uses UDP/IP as the transport mechanism for resource requests. However, this can be adapted for other network transports as well. The message structure itself is generic and easily extensible. The structure is shown in Fig 3. The resource manager takes the following kinds of requests from clients.

- QoS requests: Clients provide the resource manager with the quality of service desired for their particular application. The resource manager uses this information to influence the routing module. This information can be provided to the resource manager in a standard way. The standard IP header has a type-of-service (TOS) field that is rarely used [5]. There are four bits available to specify service types. The bits represent minimize delay, maximize throughput, maximize reliability, and minimize monetary cost. Only one of these four bits can be turned on. Applications can set these fields using socket options. Since all packets to the exterior pass through the vehicle's packet routing system, the TOS field for each packet can be examined by the vehicle's gateway router and routed appropriately.

- Requests for Communication Services: The resource manager can let the clients access common devices such as the Television or the AM/FM radio.

- Subscriptions to Information Services: The resource manager also provides indirect access to subscription services. The interface to the resource manager provides the core functions for a publisher/subscriber model.

\section{C.1 Packet Structure}

The generic packet structure for communicating with the resource manager has several fields.
- Network Header: This is the TCP/IP layer header placed by the operating system and not directly controlled by the resource manager.

- Version: A version field allows future changes to the packet structure while maintaining backward compatibility.

- Connection Identifier: Each request/response sequence is assigned a unique connection identifer by the client so that multiple responses can be associated with their respective requests.

- Request Type: This field identifies the category of the request. For example, any request associated with operating the cellular phone may have a specific predefined request type.

- Request Subtype: The value of this field specifies the exact operation to be performed. For example, dialing a number will be associated with a specific request subtype. - Length: The length field contains the length of the following data field in bytes.

- Request Specific Data: Any data that is associated with a request or response is placed in this field. It has variable length. The actual content of this field depends on the specific request or response.

\section{Design Goals}

The design of the resource manager was influenced by the following goals:

- Flexible Interface: The interface to the resource manager is always made through a network connection. The format of the requests and responses are standardized. Defining the interface as a network connection makes the interface to the resource manager programming language independent and flexible. The inner structure of the resource manager can be changed over time without any modification to clients.

- Minimal Change to Existing Applications: Software agents can provide QoS hints to the resource manager. This will allow the resource manager to provide the best external connection subject to constraints. However, standard pre-written network applications that do not know about the existence of the resource manager should work without modification. 


\section{E. Future Additions}

The current architecture will easily allow the addition of devices in the future. Future vehicle buses will be standardized and allow access to vehicle status, diagnostic information, and other systems. This will aid in remote diagnostics, automatic maintenance scheduling, and future telematics applications. Adding a vehicle bus interface to the current system will not require any substantial modification.

The developing Automotive Multimedia Interface Collaboration (AMI-C)has suggested multiple physical layer network transports for communication with the vehicle status and diagnostic systems in automobiles [6]. IEEE 1394 (FireWire) and the CAN bus are currently under consideration as physical layers and Bluetooth is a target as a future wireless interface. The current hardware architecture should support any of these network choices.

The proliferation of the Internet Protocol (IP) as the network layer of choice requires several additions for the optimal use of the QoS based routing. In particular consider a scenario where a web-browsing application on the vehicle uses an "optimal" network interface to reach a web-server. The data returned by the web-server follows a path that is not chosen by the application on the vehicle and may not be the "optimal" return path. The use of other Internet Standards such as Mobile IP [7] have to be used to correct this situation.

\section{SUMmary}

In future advanced telematics applications, various software agents will contend for the communications resources available on the vehicle. A communications resource management scheme has been proposed to coordinate the allocation of these resources. The resource manager is structured so that it can be improved without requiring modifications to the clients, and is transparent to applications not designed to use its capabilities.

\section{ACKNOWLEDGEMENTS}

The OFDM modem used in the testbed was provided by Wi-LAN, Inc. This work was supported by General Motors through the CMU/GM satellite research laboratory.

\section{REFERENCES}

[1] R. Lind, R. Schumacher, R. Reger, R. Olney, H. Yen, M. Laur, and R. Freeman, "The Network Vehicle- A Glimpse into the Future of Mobile Multi-Media," IEEE Aerospace and Electronics Systems Magazine, vol. 14, pp. 27-32, Sep 1999.

[2] J. H. Rillings and R. J. Betsold, "Advanced Driver Information Systems," IEEE Transactions on Vehicular Technology, vol. 40, pp. 31-40, Feb 1991.
[3] B. Kamali, "Some Applications of Wireless Communications in Intelligent Vehicle Highway Systems," in Vehicular Technology Conference, vol. 3, pp. 1796-1800, IEEE, Apr 1996.

[4] R. Rajkumar, M. Gagliardi, and L. Sha, "The Real-Time Publisher/Subscriber Inter-Process Communication Model for Distributed Real-Time Systems: Design and Implementation," in Real-Time Technology and Applications Symposium, pp. 66-75, May 1995.

[5] P. Almquist, "RFC 1349: Type of service in the Internet Protocol suite," July 1992.

[6] "AMI-C Architecture Specification-Release 1," tech. rep., Automotive Multimedia Interface-Collaboration, Inc., http://www.amic.org, 2000.

[7] C. Perkins, "RFC 2002: IP mobility support," Oct. 1996. 